\title{
Association between changes in lumbar Modic changes and low back symptoms over a two-year period
}

Jyri Järvinen ${ }^{1}$, Jaro Karppinen ${ }^{2,3,4^{*}}$, Jaakko Niinimäki ${ }^{1,3}$, Marianne Haapea ${ }^{1}$, Mats Grönblad ${ }^{5}$, Katariina Luoma ${ }^{6}$ and Eeva Rinne ${ }^{5}$

\begin{abstract}
Background: The association of Modic changes (MC) with low back pain (LBP) is unclear. The purpose of our study was to investigate the associations between the extent of Type 1 (M1) and Type 2 (M2) MC and low back symptoms over a two-year period.

Methods: The subjects ( $n=64$, mean age 43.8 y; 55 [86\%] women) were consecutive chronic LBP patients who had M1 or mixed M1/M2 on lumbar spine magnetic resonance imaging (MRI). Size and type of MC on sagittal lumbar MRI and clinical data regarding low back symptoms were recorded at baseline and two-year follow-up. The size (\%) of each $M C$ in relation to vertebral size was estimated from sagittal slices (midsagittal and left and right quarter), while proportions of $\mathrm{M} 1$ and $\mathrm{M} 2$ within the $\mathrm{MC}$ were evaluated from three separate slices covering the MC. The extent (\%) of $M 1$ and $M 2$ was calculated as a product of the size of $M C$ and the proportions of $M 1$ and $M 2$ within the $M C$, respectively. Changes in the extent of $M 1$ and $M 2$ were analysed for associations with changes in LBP intensity and the Oswestry disability index (ODI), using linear regression analysis.
\end{abstract}

Results: At baseline, the mean LBP intensity was 6.5 and the mean ODI was 33\%. During follow-up, LBP intensity increased in 15 patients and decreased in 41, while ODI increased in 19 patients and decreased in 44. In univariate analyses, change in the extent of M1 associated significantly positively with changes in LBP intensity and ODI (beta $0.26, p=0.036$ and beta $0.30, p=0.017$; respectively), whereas the change in the extent of M2 did not associate with changes in LBP intensity and ODI (beta $-0.24, p=0.054$ and beta $-0.13, p=0.306$; respectively). After adjustment for age, gender, and size of $\mathrm{MC}$ at baseline, change in the extent of $\mathrm{M} 1$ remained significantly positively associated with change in ODI (beta 0.53, $p=0.003$ ).

Conclusion: Change in the extent of M1 associated positively with changes in low back symptoms.

Keywords: Modic changes, Prospective study, Low back pain, Change of symptoms

\section{Background}

Modic changes (MC) are vertebral subchondral bone marrow changes that are visible in magnetic resonance imaging (MRI). They are strongly associated with degenerative disc disease [1]. MC, especially Type $1 \mathrm{MC}$ (M1), have been correlated with low back pain (LBP) in both population-based and clinical samples [2-6].

\footnotetext{
* Correspondence: jaro.karppinen@ttl.fi

${ }^{2}$ Center for Life Course Epidemiology and Systems Medicine, Faculty of

Medicine, University of Oulu, Oulu, Finland

${ }^{3}$ Medical Research Center Oulu, University of Oulu and Oulu University

Hospital, Oulu, Finland

Full list of author information is available at the end of the article
}

Three main types of MC have been described. M1 shows decreased signal intensity on T1-weighted images (T1w) and increased signal intensity on T2-weighted images (T2w) [1]. M1 is thought to represent acute inflammatory changes in degenerative disc disease, on the basis of fibrovascular replacement in histopathological specimens of subchondral bone marrow [1,2]. It has been suggested that M1 may predict a fast-progressing and deforming type of disc degeneration [7]. M1 has also been linked to an inflammatory pain pattern in clinical contexts [8]. Type $2 \mathrm{MC}$ (M2) shows increased signal intensity on both $\mathrm{T} 1 \mathrm{w}$ and $\mathrm{T} 2 \mathrm{w}$, and it appears as yellow 
marrow replacement in histopathological specimens. M2 could represent a more stable phase of degenerative disc disease, but it does have the potential to convert to another type [9-13]. Type $3 \mathrm{MC}$ (M3) shows decreased signal intensity on both $\mathrm{T} 1 \mathrm{w}$ and $\mathrm{T} 2 \mathrm{w}$ and is associated with extensive subchondral bone sclerosis on plain radiographs $[1,14,15]$. Mixed Modic types are thought to develop when one Modic type converts to another [16].

Only a few follow-up studies have evaluated the role of $\mathrm{MC}$ types and $\mathrm{MC}$ conversions in relation to low back symptoms [5,17]. The presence of M1 at both baseline and 14-month follow-up was found to be associated with poor outcome in patients with persistent LBP and MC [5]. Moreover, it has been suggested that as M1 converts to $\mathrm{M} 2$, pain intensity and perceived disability subside [18]. The aim of this study was to investigate associations between changes in the size and type of $\mathrm{MC}$ and low back symptoms over a two-year follow-up.

\section{Methods}

\section{Study population}

The study population was selected from consecutive LBP patients $(n=4380)$ with or without radicular symptoms who were referred initially for standard lumbar spine MRI to the Departments of Orthopaedics, Rheumatology or Physical and Rehabilitation Medicine at the region of Helsinki University Hospital during 2003-2007. Images from all patients examined by MRI were analysed monthly, and eligible patients were identified by an experienced radiologist. The inclusion criteria were chronic nonspecific LBP of at least three-month duration and lumbar M1 or mixed M1/M2 [18]. All included patients gave written informed consent to use their clinical data for study purposes. The study protocol was approved by the Ethics Committee of Helsinki and the Uudenmaa District University Hospitals.

The exclusion criteria were age $\geq 65 \mathrm{y}$; specific back disease, such as fracture, neoplasia, infectious, or rheumatic spine disease; spondylolisthesis ( $\geq 4 \mathrm{~mm})$; spinal stenosis; disc extrusion; any other finding with even the slightest neural compression; minor spine operation, such as herniated disc surgery within the past six months; and major spine operation, such as fusion or disc prosthesis at any time. Annular tears, bulging of the disc, and facet joint degeneration were not exclusion criteria, since these changes are often found in association with disc degeneration and also with MC. When there was uncertainty about the etiology of signal abnormalities, we checked laboratory results and other clinical findings to exclude specific causes (e.g., infectious or rheumatic spinal disease).

Within 1-3 weeks of identification, eligible patients were contacted by telephone to complete questionnaires to describe average LBP intensity during the past week (scale $0-10 ; 0=$ no pain, $10=$ worst pain possible) and obtain the Oswestry Disability Index (ODI, version 1.0; scale $0-100 \%: 0 \%=$ no disability, $100 \%=$ very severe disability). ODI was obtained by a patient-completed questionnaire that generates a subjective percentage score of level of function (disability) in activities of daily living among back pain sufferers [19]. The time interval between baseline MRI and symptom assessment varied from two to six weeks. Standard lumbar spine MRI was performed again at the two-year follow-up visit, 23-25 months after baseline imaging. Questionnaires for average LBP intensity and ODI were completed during the follow-up visit.

\section{Imaging methods}

The MRI studies at baseline were performed with two $1.0 \mathrm{~T}$ (Gyroscan Intera, Philips Medical Systems, Eindhoven, The Netherlands) and three $1.5 \mathrm{~T}$ (Signa HD, GE Healthcare, Milwaukee, WI, USA and Sonata and Symphony, Siemens Medical, Erlangen, Germany) units using the established spine imaging protocols of the participating hospitals. The imaging parameters of $\mathrm{T} 1$ - and T2-weighted turbo spin-echo (TSE) or fast spin-echo (FSE) sequences were conventional: for example, $13 \mathrm{~ms}$ TE and $600 \mathrm{~ms}$ TR (short TE and TR) for T1w and $115 \mathrm{~ms}$ TE and $4000 \mathrm{~ms}$ TR (long TE and TR) for T2w. At follow-up, all MRI images were obtained with a $1.0 \mathrm{~T}$ unit (Gyroscan Intera, Philips Medical Systems), following a uniform protocol [7].

\section{Image analysis}

Evaluations of the baseline and two-year follow-up images were performed by visually examining hard copies of sagittal T1WIs and T2WIs. We chose visual analysis of hard copies for uniformity of assessments, as all participating hospitals did not have digital picture archiving and communication systems (PACS) at the beginning of data collection. All images were assessed by a fellow in musculoskeletal radiology (JJ) who was blinded to the patients' symptoms. To estimate the interobserver reliability, an experienced musculoskeletal radiologist (JN) evaluated images of 30 endplates from randomly selected patients.

Each patient's baseline and follow-up MR images were assessed on an x-ray light box, starting with the baseline images. Removal of imaging dates during evaluation of the images was not considered necessary, because the reader was blinded to clinical data. The relative size in percentages (intervals of 5\%) of each MC compared to corresponding vertebra in sagittal images was estimated as the average of assessments of three slices (midsagittal and left and right quarter) from T2w. Next, the proportions (\%) of M1 and M2 within the MC were estimated from three slices (middle and left and right quarter) covering MC of sagittal T1w and T2w. 


\section{Statistical analysis}

Descriptive statistics were calculated to describe the data. Reader reliability was assessed using intraclass correlation coefficients (ICC; absolute agreement). ICC can be interpreted as follows: $<0.40 \%$ poor, $0.40-0.59$ fair, 0.60-0.74 good, and 0.75-1.00 excellent [20]. Limits of agreement were also calculated. The extent of M1 and M2 at baseline and follow-up were calculated by multiplying the size of $\mathrm{MC}$ at both endplates by the corresponding proportions of M1 and M2, respectively, and summing up the products of both endplates. Only M1 and M2 were used in the analyses, due to the low prevalence of M3 (Table 1). Changes in the extent of M1 and M2 over the follow-up were calculated, as well as changes in low back symptoms. Linear regression analysis was used to evaluate the association between changes in the extent of M1 and M2 and low back symptoms, both unadjusted and adjusted for age, gender, and size of MC at baseline. IBM SPSS Statistics version 22 was used in the analyses.

\section{Results}

\section{Study population}

The baseline study population consisted of 75 chronic LBP patients ( $87 \%$ women) with M1 in the lumbar spine. During follow-up, 11 patients were lost as dropouts: seven due to lack of clinical data and four who were not scanned at follow-up. In all, 64 patients (86\% women) were available for the final analyses. Mean age at baseline was 43.8 y (standard deviation [SD] 9.8, range 2464 y).

\section{Reliability of image reading}

Reliability between the two readers for evaluation of size of MC was excellent (ICC 0.80). Reliability of the evaluation of proportions of M1 and M2 within the observed

Table 1 Size and proportion of Modic Type 1 (M1), Type 2 (M2) and Type 3 (M3) at baseline and follow-up, and scores of low back symptoms at baseline and follow-up

\begin{tabular}{lll}
\hline & $\begin{array}{l}\text { Baseline } \\
(\mathbf{n}=\mathbf{1 2 4})^{\mathbf{2}}\end{array}$ & $\begin{array}{l}\text { Follow-up } \\
\text { (n= } \mathbf{n} \text { 126) }\end{array}$ \\
& $20.7(12.3)$ & $24.4(12.6)$ \\
\hline $\begin{array}{l}\text { Mean (SD) } \\
\text { Proportion (\%) of }\end{array}$ & & \\
M1 & $74.2(26.2)$ & $40.6(31.1)$ \\
M2 & $23.9(26.2)$ & $56.2(30.2)$ \\
M3 & $1.9(5.3)$ & $3.2(8.8)$ \\
Low back symptoms & & \\
Low back pain intensity (0-10) & $6.5(1.9)$ & $5.2(2.7)$ \\
Oswestry Disability Index (0-100\%) & $33.2(14.4)$ & $28.1(19.0)$ \\
\hline
\end{tabular}

${ }^{1} \%$ from vertebral volume.

${ }^{2}$ Endplates with Modic changes.
MC was also excellent (ICC 0.85 and 0.93 for M1 and M2, respectively). Limits of agreement ranged from -10 to 17 (mean difference between JJ and JN 3.4, SD 6.8) for the size of MC, from -44 to 27 (mean -8.8, SD 18.1) for the proportion of M1, and from -20 to 31 (mean 5.6, SD 12.9) for the proportion of M2.

\section{MRI findings}

Most MCs were located at L4/5 or L5/S1 (39\% and 49\%, respectively, at baseline). The mean size of the $\mathrm{MC}$ in relation to vertebral size at baseline was $21 \%$ (SD 12, range 5-55\%). At the two-year follow-up, the mean size was $24 \%$ (SD 13, range 7.5-60\%). The mean proportion of the M1 component within the MC was $74 \%$ at baseline and $41 \%$ at follow-up, while the mean proportion of the M2 component was 24\% at baseline and 56\% at follow-up (Table 1).

\section{Clinical symptoms}

At baseline, the mean LBP intensity was 6.5 (SD 1.9, range 1-10) and ODI was 33\% (SD 14, range 8-66). At follow-up, the mean LBP intensity was 5.2 (SD 2.7, range $0-9$ ) and ODI was 28\% (SD 19, range 0-78; Table 1). Also at follow-up, the intensity of LBP had increased in 15 patients (23\%; mean 2.5, SD 1.5) and decreased in 41 patients (64\%; mean -3.0 , SD 2.0), while the ODI had increased in 19 patients (30\%, mean $13.7 \%$, SD 9.8) and decreased in 44 patients $(69 \%$, mean $-13.4 \%$, SD 9.1) (Figures 1 and 2).

\section{Association between MRI findings and clinical symptoms} Change in the extent of M1 associated positively with changes in LBP intensity and ODI (beta $0.26, p=0.036$ and beta $0.30, p=0.017$; respectively, whereas change in the extent of M2 associated negatively with changes in LBP intensity and ODI (Table 2, Figures 3 and 4). However, this latter association was not statistically significant. When adjusted for age, gender, and size of $\mathrm{MC}$ at baseline, the association between the change in the extent of M1 and LBP intensity became non-significant, whereas the association between the change in the extent of M1 and ODI remained significant (Table 2).

\section{Discussion}

In this two-year follow-up study, we found significant positive associations between the change in the extent of M1 and changes in both LBP intensity and ODI. The association between changes in M1 and ODI remained significant after adjustment for confounders. Change in the extent of M2 had non-significant negative associations with changes in LBP intensity and ODI. The study sample was chosen to represent patients with M1 in the lumbar spine. The proportion of M1 within MC was $73 \%$ on average at baseline. In most patients, the 

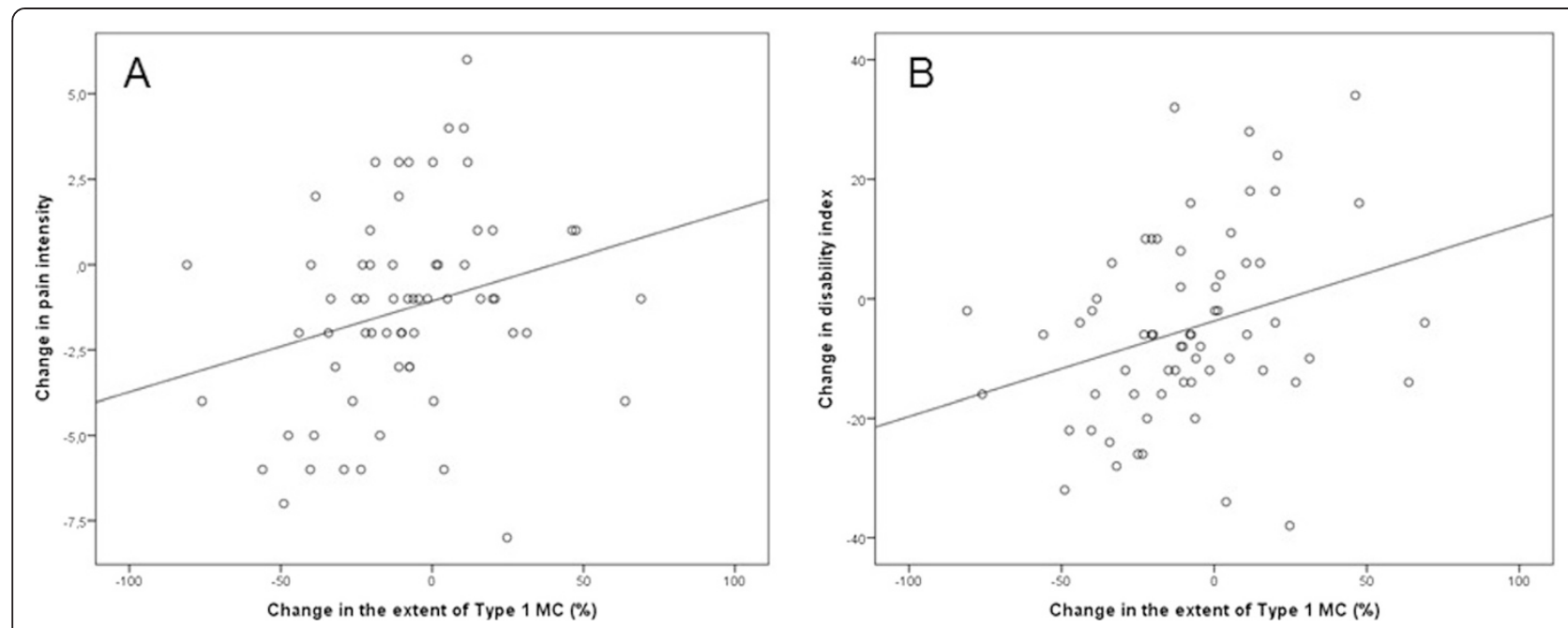

Figure 1 Scatter boxes showing (A) the positive correlation between change in the extent of Type 1 Modic change and change in low back pain intensity and (B) the positive correlation between change in the extent of Type 1 Modic change and change in Oswestry Disability Index.

proportion of M1 within MC decreased during follow-up, while the proportion of M2 increased. Both LBP intensity and ODI were more likely to decrease than increase during follow-up. For a 50\% decrease in the extent of M1, LBP intensity decreased 2.4 units in VAS, and disability decreased 11.7 units in ODI, while for a 10\% decrease the corresponding estimates were -1.3 in VAS and -5.3 in ODI.

It is estimated that $<15 \%$ of patients who seek care for LBP have symptoms due to a specific cause (e.g., fracture, infection, tumour, or compression of the nerve root). The vast majority of LBP patients are thus classified as having nonspecific LBP. Recently, there has been growing interest in identifying and classifying LBP according to specific clinical subgroups [21]. In some studies, LBP patients with MC have been suggested to be a specific subgroup of LBP [2,22-24]. In a recent prospective cohort study [6], the authors sampled 140 patients to investigate associations between baseline degenerative imaging findings and outcome in sick-listed LBP patients. They concluded that M1 was the only degenerative finding that predicted persistent symptoms and sick leaves. However, the association between MC and LBP remains debatable [25-27].

MC have not only been observed among patients with LBP $[4,28]$, but also in MR images obtained in populationbased studies $[9,29]$ and even in asymptomatic subjects [30-32]. Only a few follow-up studies have investigated the

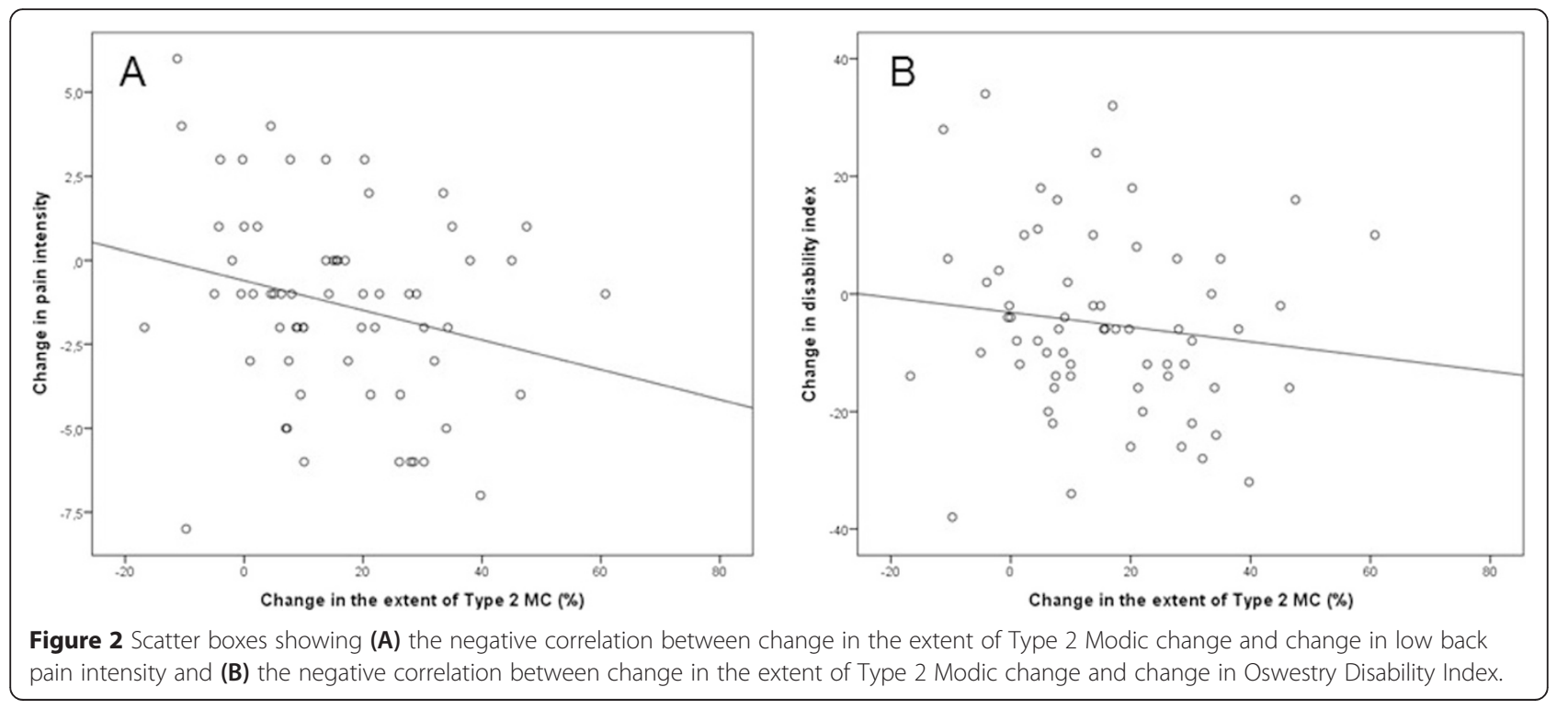


Table 2 Association of change in the extent of Modic Type 1 (M1) and Type 2 (M2) with low back pain (LBP) intensity and Oswestry Disability Index (ODI)

\begin{tabular}{|c|c|c|c|c|c|c|}
\hline & \multicolumn{3}{|l|}{ LBP intensity } & \multicolumn{3}{|l|}{ ODI } \\
\hline & $\mathrm{B}(\mathrm{SE})$ & Beta & $\mathbf{P}$ & $\mathrm{B}(\mathrm{SE})$ & Beta & $\mathbf{P}$ \\
\hline \multicolumn{7}{|l|}{ Unadjusted } \\
\hline Change in M1 & $0.027(0.012)$ & 0.26 & 0.036 & $0.160(0.065)$ & 0.30 & 0.017 \\
\hline Change in $\mathrm{M} 2$ & $-0.044(0.022)$ & -0.24 & 0.054 & $-0.125(0.122)$ & -0.13 & 0.306 \\
\hline \multicolumn{7}{|c|}{ Adjusted for age and gender } \\
\hline Change in $\mathrm{M} 1$ & $0.025(0.013)$ & 0.25 & 0.060 & $0.166(0.068)$ & 0.31 & 0.018 \\
\hline Change in $\mathrm{M} 2$ & $-0.044(0.023)$ & -0.24 & 0.059 & $-0.128(0.124)$ & -0.13 & 0.304 \\
\hline \multicolumn{7}{|c|}{ Adjusted for age, gender and size of Modic change at baseline } \\
\hline Change in M1 & $0.019(0.018)$ & 0.19 & 0.284 & $0.282(0.090)$ & 0.53 & 0.003 \\
\hline Change in $\mathrm{M} 2$ & $-0.035(0.024)$ & -0.19 & 0.156 & $-0.135(0.133)$ & -0.14 & 0.315 \\
\hline
\end{tabular}

associations between low back symptoms and M1 [5,17]. A Danish follow-up cohort study investigated the development of $\mathrm{MC}$ over a 14-month period and whether changes in the size and type of $\mathrm{MC}$ were associated with changes in clinical symptoms [5]. They concluded that patients with M1 at both baseline and follow-up had a poor prognosis compared to those without M1 at baseline and follow-up. Their results are in accordance with our findings. Moreover, their study design resembles ours, and although our study sample was smaller, we had a longer follow-up. In a longitudinal study by Mitra et al. [17], the authors found a trend of higher pain intensity and disability scores among patients with an increase in the M1 component and lower scores in patients with conversion of M1 to M2.
Our results suggest a statistically significant association between M1 and low back symptoms; even after adjustments, the associations between changes in the extent of M1 and ODI were significant. Hence, our findings support the observation that M1 has a stronger association with LBP than other types of MC [4,16-18,29,33,34]. In a study by Kääpä et al. [18], patients with chronic LBP and M1 suffered from pain and disability significantly more than patients with mixed type M1/2 changes. The authors' tentative interpretation was that as M1 converts to M2, pain intensity and perceived disability subside. Our finding, which concerned mainly the same study population as that in [18], is in accordance with this interpretation, although a significant association with symptoms was
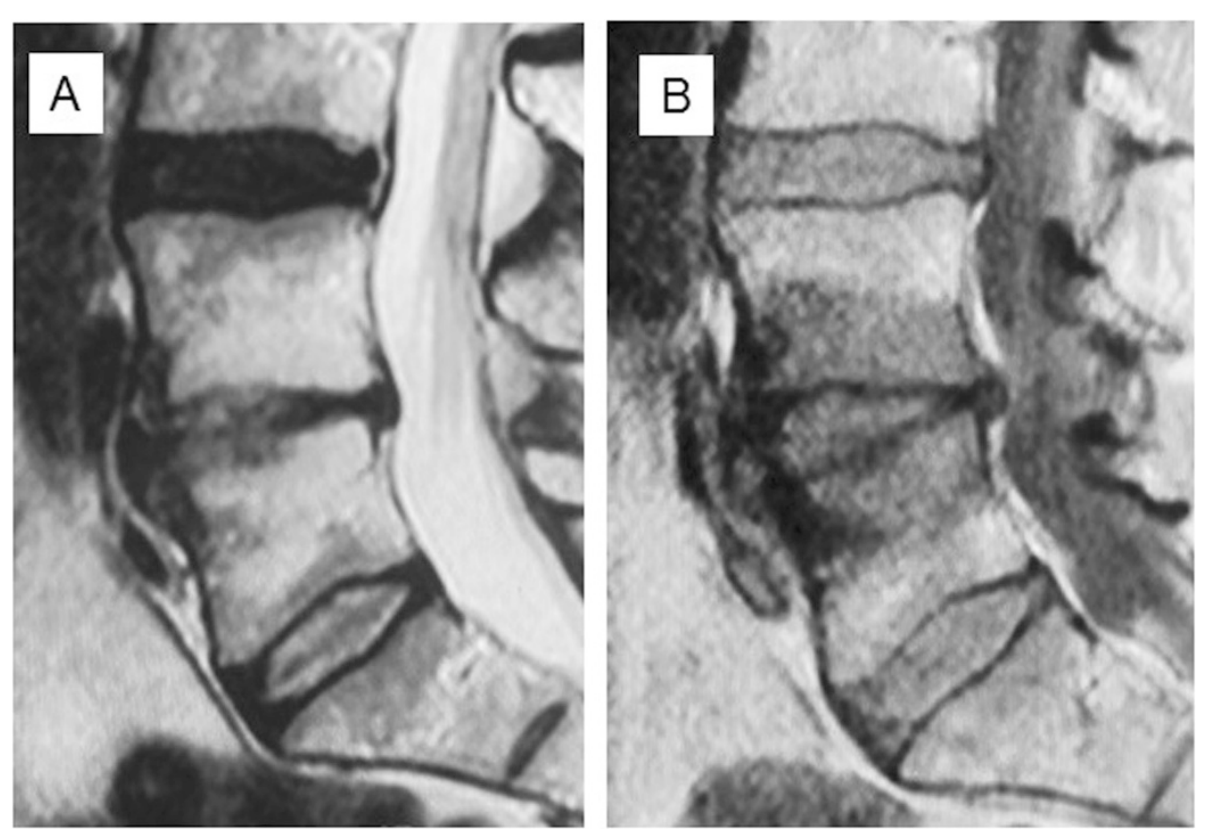

Figure 3 Baseline T2 (A) and T1 (B) -weighted sagittal MR images of a 45-year-old man with Type 1 Modic change at L4/5. Low back pain intensity 8/10, Oswestry Disability Index 24\%. 

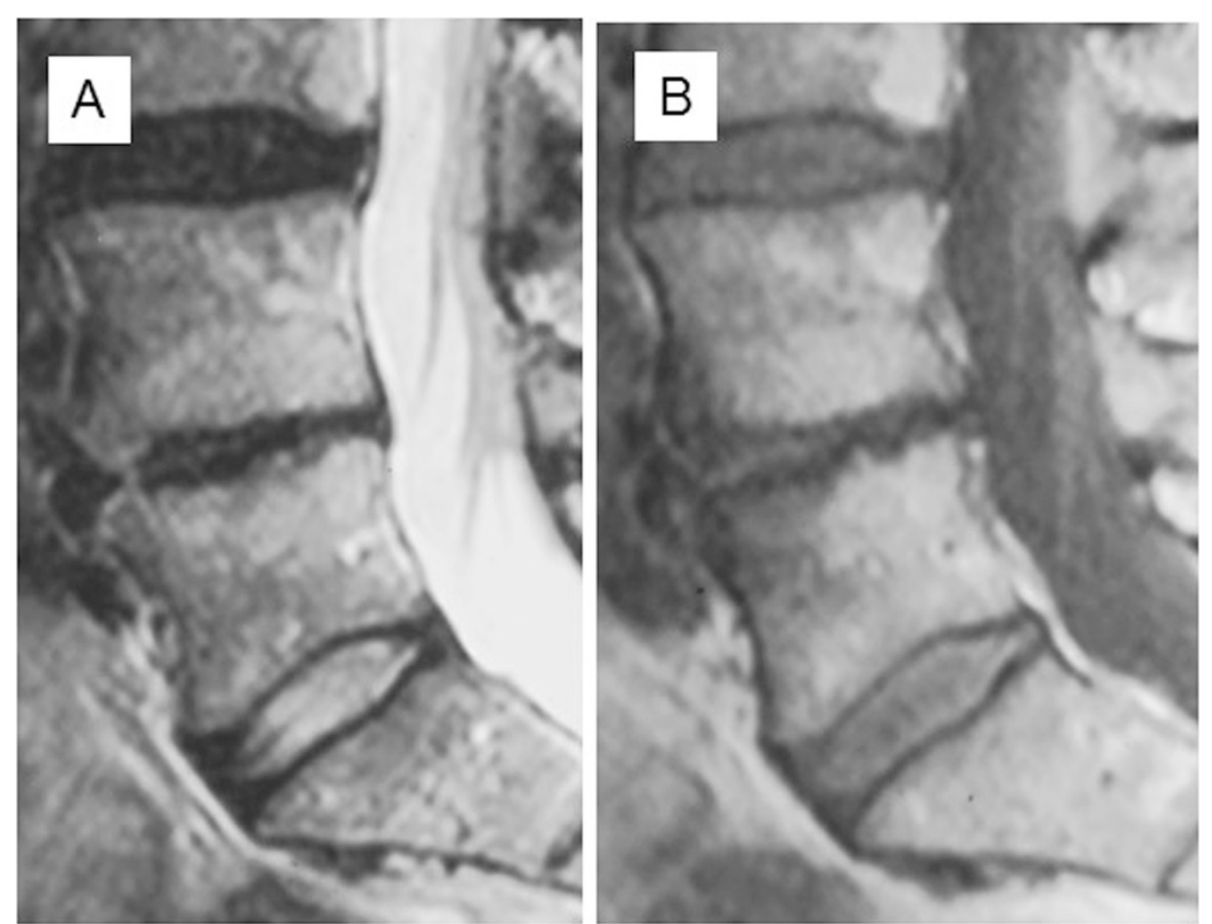

Figure 4 T2 (A) and T1 (B) -weighted sagittal MR images at follow-up of the same patient as in Figure 1 showing that the Modic change has converted to Type 2. Low back pain intensity 2/10, Oswestry Disability Index 12\%.

observed only for changes in the extent of M1, not for changes in the extent of M2. In a Finnish study [4], the authors compared self-reported LBP and Modic findings on MRI in a sample of middle-aged male workers. Altogether, $178 \mathrm{MCs}$ in 128 subjects were recorded: $30 \% \mathrm{M} 1,66 \% \mathrm{M} 2$, and $4 \%$ both M1 and M2. They concluded that MC at L5/S1 and M1 lesions were more likely to be associated with pain symptoms than other types of MC, or MC located at other lumbar levels. A study of chronic LBP patients by Kerttula et al. [7] indicated that even during the one-year follow-up, both increasing and decreasing M1 changes were associated with an accelerated process of adjacent disc degeneration, while disc degeneration in the absence of M1 seemed to advance more slowly. M1 may signify a distinct degenerative process in the discovertebral unit [11].

Only two randomized trials have evaluated the efficacy of medication for LBP due to MCs. In a Danish study [35], amoxicillin-clavulanate treatment for three months was effective compared to placebo among patients with M1 after verified disc herniation. In another study [36], zoledronic acid, a long-acting bisphosphonate, was effective in reducing the intensity of LBP in the short-term and in reducing the use of nonsteroidal anti-inflammatory drugs at oneyear follow-up among patients with chronic LBP and MC confirmed by MRI. Although these results are promising, more research must be carried out to replicate both treatment interventions.

There are a few limitations that should be considered in our study. First, the study population is quite small. However, solitary M1 or mixed M1/M2 is uncommon, which complicated patient recruitment. Furthermore, we excluded potential clinically relevant changes from other disc levels. Therefore, the patients needed to be collected from several hospitals that used non-identical MR equipment. Differing MR equipment may influence image quality, but uniform imaging protocols were used for high-field MRI. In a Danish study [37], the authors compared lumbar MC in low-field (0.3 T) MRI and high-field (1.5 T) scanners. They concluded that there was a difference between lowand high-field MRI in terms of overall prevalence of MC; the number of MC diagnosed with high-field MRI was significantly higher than with low-field MRI. M1 dominated in low-field scanners, and M2 in high-field scanners. Because differences between the field strengths of our equipment were minor (1.0-1.5 T), we believe that the different scanners should not have a marked influence on the assessment of type or size of MC. The potential influence on LBP symptoms of other degenerative imaging findings (e.g., annular tears and disc bulging), pain medication and other treatments, somatic and psychological comorbidities, educational level, compensation, and other psychosocial elements was not analyzed. 


\section{Conclusions}

In this 2-year follow-up study, we found a significant positive association between the change in the extent of M1 and change in ODI. We also found a positive association between the change in the extent of M1 and LBP intensity, which, however, became non-significant after the adjustments. These results lend support to the hypothesis that LBP patients with M1 represent a specific subgroup of patients with LBP.

\section{Competing interests}

The authors declare that they have no competing interests.

\section{Authors' contributions}

JJ designed the research protocol together with JK and ER; ER, KL and MG collected the study population; JJ and JN made all measurements; JJ had most significant role in drafting manuscript; $\mathrm{MH}$ performed the statistical analyses. All authors helped to draft the manuscript, and read and approved the final manuscript.

\section{Acknowledgements}

We are grateful to Helsinki University Central Hospital for being able to perform the study.

\section{Author details}

${ }^{1}$ Department of Diagnostic Radiology, Institute of Diagnostics, Oulu University Hospital, Oulu, Finland. ${ }^{2}$ Center for Life Course Epidemiology and Systems Medicine, Faculty of Medicine, University of Oulu, Oulu, Finland. ${ }^{3}$ Medical Research Center Oulu, University of Oulu and Oulu University Hospital, Oulu, Finland. ${ }^{4}$ Finnish Institute of Occupational Health, Health and Work Ability, and Disability Prevention Centre, Oulu, Finland. ${ }^{5}$ Department of Physical and Rehabilitation Medicine, Institute of Clinical Sciences, University of Helsinki, Helsinki, Finland. 'University of Helsinki and HUS Imaging Center, Helsinki University Central Hospital, Helsinki, Finland.

Received: 8 October 2014 Accepted: 27 March 2015

Published online: 22 April 2015

\section{References}

1. Modic MT, Steinberg PM, Ross JS, Masaryk TJ, Carter JR. Degenerative disk disease: Assessment of changes in vertebral body marrow with MR imaging. Radiology. 1988;166:193-9.

2. Kjaer P, Korsholm L, Bendix T, Sørensen JS, Leboeuf-Yde C. Modic changes and their associations with clinical findings. Eur Spine J. 2006;15:1312-9.

3. Jensen TS, Karppinen J, Sørensen JS, Niinimäki J, Leboeuf-Yde C. Vertebral endplate signal changes (Modic change): A systematic literature review of prevalence and association with non-specific low back pain. Eur Spine J. 2008;17:1407-22.

4. Kuisma M, Karppinen J, Niinimäki J, Ojala R, Haapea M, Heliövaara M, et al. Modic changes in endplates of lumbar vertebral bodies: Prevalence and association with low back and sciatic pain among middle-aged male workers. Spine (Phila Pa 1976). 2007;32:1116-22.

5. Jensen RK, Leboeuf-Yde C, Wedderkopp N, Sørensen JS, Jensen TS, Manniche C. Is the development of modic changes associated with clinical symptoms? A 14-month cohort study with MRI. Eur Spine J. 2012;21:2271-9.

6. Jensen OK, Nielsen CV, Sørensen JS, Stengaard-Pedersen K. Type 1 Modic changes was a significant risk factor for 1 year outcome in sick-listed low back pain patients: a nested cohort study using magnetic resonance imaging of the lumbar spine. Spine J 2014, doi:10.1016/j.spinee.2014.02.018. [Epub ahead of print]

7. Kerttula L, Luoma K, Vehmas T, Grönblad M, Kääpä E. Modic type I change may predict rapid progressive, deforming disc degeneration: A prospective 1-year follow-up study. Eur Spine J. 2012;21:1135-42.

8. Bailly F, Maigne JY, Genevay S, Marty M, Gandjbakhch F, Rozenberg S, et al Inflammatory pain pattern and pain with lumbar extension associated with Modic 1 changes on MRI: a prospective case-control study of 120 patients. Eur Spine J. 2014;23:493-7.

9. Jensen TS, Bendix T, Sørensen JS, Manniche C, Korsholm L, Kjaer P. Characteristics and natural course of vertebral endplate signal (Modic) changes in the Danish general population. BMC Musculoskelet Disord. 2009;10:81.

10. Luoma K, Vehmas T, Grönblad M, Kerttula L, Kääpä E. MRI follow-up of subchondral signal abnormalities in a selected group of chronic low back pain patients. Eur Spine J. 2008;17:1300-8.

11. Luoma K, Vehmas T, Grönblad M, Kerttula L, Kääpä E. Relationship of Modic type 1 change with disc degeneration: A prospective MRI study. Skeletal Radiol. 2009;38:237-44

12. Kuisma M, Karppinen J, Niinimäki J, Kurunlahti M, Haapea M, Vanharanta H, et al. A three-year follow-up of lumbar spine endplate (Modic) changes. Spine (Phila Pa 1976). 2006;31:1714-8.

13. Marshman LA, Trewhella M, Friesem T, Bhatia CK, Krishna M. Reverse transformation of Modic type 2 changes to Modic type 1 changes during sustained chronic low-back pain severity. Report of two cases and review of the literature. J Neurosurg Spine. 2007;6:152-5.

14. Modic MT, Masaryk TJ, Ross JS, Carter JR. Imaging of degenerative disk disease. Radiology. 1988;168:177-86.

15. de Roos A, Kressel H, Spritzer C, Dalinka M. MR imaging of marrow changes adjacent to end plates in degenerative lumbar disk disease. AJR Am J Roentgenol. 1987;149:531-4.

16. Vital JM, Gille O, Pointillart V, Pedram M, Bacon P, Razanabola F, et al. Course of Modic 1 six months after lumbar posterior osteosynthesis. Spine (Phila Pa 1976). 2003;28:715-20.

17. Mitra D, Cassar-Pullicino VN, McCall IW. Longitudinal study of vertebral type-1 end-plate changes on MR of the lumbar spine. Eur Radiol. 2004;14:1574-81.

18. Kääpä E, Luoma K, Pitkäniemi J, Kerttula L, Grönblad M. Correlation of size and type of modic type 1 and 2 lesion with clinical symptoms - a descriptive study in a subgroup of chronic low back pain patients based on a university hospital patient sample. Spine (Phila Pa 1976). 2012;37:134-9.

19. Fairbank JC, Couper J, Davies JB, O'Brien JP. The Oswestry low back pain disability questionnaire. Physiotherapy. 1980;66:271-3.

20. Cicchetti D, Bronen R, Spencer S, Haut S, Berg A, Oliver P, et al. Rating scales, scales of measurement, issues of reliability: resolving some critical issues for clinicians and researchers. J Nerv Ment Dis. 2006;194:557-64.

21. Airaksinen O, Brox JI, Cedraschi C, Hildebrandt J, Klaber-Moffett J, Kovacs F, et al. COST B13 Working Group on Guidelines for Chronic Low Back Pain. Chapter 4. European guidelines for the management of chronic nonspecific low back pain. Eur Spine J. 2006;15 Suppl 2:S192-300.

22. Albert HB, Kjaer P, Jensen TS, Sørensen JS, Bendix T, Manniche C. Modic changes, possible causes and relation to low back pain. Med Hypotheses. 2008;70:361-8

23. Albert HB, Manniche C. Modic changes following lumbar disc herniation. Eur Spine J. 2007;16:977-82

24. Jensen RK, Leboeuf-Yde C. Is the presence of modic changes associated with the outcomes of different treatments? A systematic critical review. BMC Musculoskelet Disord. 2011;12:183.

25. Kovacs FM, Arana E, Royuela A, Estemera A, Amengual G, Asenjo B. Vertebral endplate changes are not associated with chronic low back pain among Southern European subjects: A case control study. AJNR Am J Neuroradiol. 2012;33:1519-24

26. Sandhu HS, Sanchez-Caso LP, Parvataneni HK, Cammisa Jr FP, Girardi FP, Ghelman P. Association between findings of provocative discography and vertebral endplate signal changes as seen on MRI. J Spinal Disord. 2000;13:438-43.

27. el Barzouhi A, Vleggeert-Lankamp $C L$, van der Kallen BF, Nijeholt GJ L à, van den Hout WB, Koes BW, et al. Leiden-Hague Spine Intervention Prognostic Study Group. Back pain's association with vertebral end-plate signal changes in sciatica. Spine J. 2014;14:225-33.

28. Rahme R, Moussa R. The modic vertebral endplate and marrow changes: Pathologic significance and relation to low back pain and segmental instability of the lumbar spine. AJNR Am J Neuroradiol. 2008;29:838-42.

29. Kjaer P, Leboeuf-Yde C, Korsholm L, Sørensen JS, Bendix T. Magnetic resonance imaging and low back pain in adults: A diagnostic imaging study of 40-year-old men and women. Spine (Phila Pa 1976). 2005;30:1173-80.

30. Chung CB, Vande Berg BC, Tavernier T, Cotton A, Laredo JD, Vallee C, et al. End plate marrow changes in the asymptomatic lumbosacral spine: Frequency, distribution and correlation with age and degenerative changes. Skeletal Radiol. 2004;33:399-404.

31. Kanayama M, Togawa D, Takahashi C, Terai T, Hashimoto T. Cross-sectional magnetic resonance imaging study of lumbar disc degeneration in 200 healthy individuals. J Neurosurg Spine. 2009;11:501-7. 
32. Weishaupt D, Zanetti M, Hodler J, Min K, Fuchs B, Pfirrmann CW, et al.

Painful lumbar disk derangement: Relevance of endplate abnormalities at MR imaging. Radiology. 2001;218:420-7.

33. Toyone T, Takahashi K, Kitahara H, Yamagata M, Murakami M, Moriya H. Vertebral bone-marrow changes in degenerative lumbar disc disease. An MRI study of 74 patients with low back pain. J Bone Joint Surg (Br). 1994;76:757-64.

34. Thompson KJ, Dagher AP, Eckel TS, Clark M, Reinig JW. Modic changes on MR images as studied with provocative diskography: Clinical relevance-a retrospective study of 2457 disks. Radiology. 2009;250:849-55.

35. Albert HB, Sørensen JS, Christensen BS, Manniche C. Antibiotic treatment in patients with chronic low back pain and vertebral bone edema (Modic type 1 changes): a double-blind randomized clinical controlled trial of efficacy. Eur Spine J. 2013;22:690-6.

36. Koivisto K, Kyllönen E, Haapea M, Niinimäki J, Sundqvist K, Pehkonen T, et al. Efficacy of zoledronic acid for chronic low back pain associated with Modic changes in magnetic resonance imaging. BMC Musculoskelet Disord. 2014;15:64.

37. Bendix T, Sørensen JS, Henriksson GA, Bolstad JE, Narvestad EK, Jensen TS. Lumbar modic changes - A comparison between findings at low-and high-field MRI. Spine (Phila Pa 1976). 2012;37:1756-62

\section{Submit your next manuscript to BioMed Central and take full advantage of:}

- Convenient online submission

- Thorough peer review

- No space constraints or color figure charges

- Immediate publication on acceptance

- Inclusion in PubMed, CAS, Scopus and Google Scholar

- Research which is freely available for redistribution 